\title{
Effect of Beta-Blockers on Stroke Outcome: A Meta-Analysis
}

This article was published in the following Dove Press journal: Clinical Epidemiology

\author{
Hajnal Zsuzsanna Balla' \\ Yang $\mathrm{Cao}^{2,3}$ \\ Jakob O Ström ${ }^{1,4}$
}

'Department of Neurology, Faculty of Medicine and Health, Örebro University, Örebro, Sweden; ${ }^{2}$ Clinical Epidemiology and Biostatistics, School of Medical Sciences, Örebro University, Örebro, Sweden; ${ }^{3}$ Unit of Biostatistics, Institute of Environmental Medicine, Karolinska Institutet, Stockholm, Sweden; ${ }^{4}$ Department of Clinical Chemistry and Department of Clinical and Experimental Medicine, Linköping University,

Linköping, Sweden
Correspondence: Jakob O Ström Department of Neurology, Faculty of Medicine and Health, Örebro University Hospital, Örebro, 70I 85, Sweden Tel +46 196021000

Email jakob.strom@oru.se
Introduction: Cardiovascular events and infections are common in the acute phase after stroke. It has been suggested that these complications may be associated with excessive sympathetic activation due to the stroke, and that beta-adrenergic antagonists (beta-blockers) therefore may be beneficial.

Aim: The aim of the current meta-analysis was to investigate the association between betablocker treatment in acute stroke and the three outcomes: mortality, functional outcome and post-stroke infections.

Methods: A literature search was performed using the keywords stroke, cerebrovascular disorders, adrenergic beta-antagonists, treatment outcome and mortality. Randomized clinical trials and observational studies were eligible for data extraction. Heterogeneity was investigated using $\mathrm{I}^{2}$ statistics. Random effect model was used when heterogeneity presented among studies; otherwise, a fixed-effect model was used. Publication bias was assessed using Egger's test and by visually inspecting funnel plots.

Results: A total of 20 studies were eligible for at least one of the three outcomes. Two of the included studies were randomized controlled trials and 18 were observational studies. Quality assessments indicated that the risk of bias was moderate. The meta-analysis found no significant association between treatment with beta-blockers and any of the three outcomes. The studies analyzed for the outcomes mortality and infection were heterogeneous, while studies analyzed for functional outcome were homogeneous. The articles analyzed for mortality showed signs of publication bias.

Conclusion: The lack of significant effects in the current meta-analysis, comprising more than 100,000 patients, does not support the proposed beneficial effects of beta-blockers in the acute phase of stroke.

Keywords: stroke, beta-blockers, mortality, outcome, infection

\section{Introduction}

Stroke and ischemic heart disease are the leading causes of death worldwide and accounted for 15.2 million deaths in 2016. Despite improvements and advances in healthcare and treatment methods, the impact on global mortality and morbidity remains high. ${ }^{1}$

A pathological sympathetic activation with a surge in catecholamines occurs in the acute phase of stroke, ${ }^{2}$ and both the sympathetic over-activation and the subsequent autonomic dysfunction are predictors of poor functional outcome. ${ }^{2,3}$ In addition to increasing the risk of cardiac arrhythmias, ${ }^{4}$ the autonomic dysfunction and sympathetic over-activation may have a role in the development of stroke-induced immunodepression, which could increase the risk of infections. ${ }^{5,6}$ 
The post-stroke changes in the immune system include an increased number of circulating monocytes, higher levels of anti-inflammatory cytokines and a shift from Th1 to Th2 cytokine production. ${ }^{6,7}$ In animal models, betablockers showed a protective effect and an ability to block and reverse these catecholamine-induced changes on the immune system. ${ }^{7,8}$ In addition to this, betablockers have been reported to reduce infarct size by antioxidant and free radical scavenging properties. ${ }^{9-11}$ However, human observational and interventional studies regarding the effects of beta-blockers in the acute face of stroke have been inconclusive and conflicting. While some have reported beneficial effects, ${ }^{12,13}$ other studies have demonstrated the opposite ${ }^{14-16}$ or been negative. ${ }^{17,18}$ Despite this obvious discordance and a relatively high number of studies investigating this important topic, no systematic review has been performed. This prompted us to perform a meta-analysis to assess whether there is an association between treatment with beta-blockers in the acute phase after stroke and stroke outcome.

\section{Materials and Methods}

This study was conducted in accordance with the Preferred Reporting Items for Systematic Reviews and Metaanalyses (PRISMA) statement, ${ }^{19}$ and was registered in the PROSPERO database (CRD42019124045).

\section{Search Strategy}

The databases PubMed, Cochrane, Medline and Scopus were searched until September 19, 2018. A combination of the following search terms was used: stroke, cerebrovascular disease, adrenergic beta-antagonists, treatment outcome and mortality. The Mesh term for each search term was searched in combination with all the English synonyms associated with the respective Mesh term. For a detailed description of each search see the Supplementary Tables S1-S4. Titles and abstracts for each database search result were screened based on the inclusion and exclusion criteria. The reference list, similar articles list in PubMed, and list of articles that cited the respective article, for each article of interest, were also hand searched. Finally, the full text of each article of interest was reviewed. One author (HSB) concluded the database searches, screened the titles and abstracts and excluded the articles not meeting the inclusion criteria.
The same author was also responsible for the full-text assessment of the eligible studies. When uncertainties arose on whether a study should be included, the senior author (JOS) was consulted.

\section{Study Selection}

The aim of the present meta-analysis was to investigate if there is an association between beta-blocker therapy and stroke outcome within the first 12 months after an acute stroke. The study design of included studies consisted of randomized clinical trials and observational studies. Studies eligible for inclusion had to include a sample of at least $90 \%$ ischemic and/or hemorrhagic stroke patients; other neurological diagnoses could be included if these did not comprise more than $10 \%$ of the study population. The intervention of interest was treatment with beta-blockers in the acute phase of stroke defined as the first 7 days from stroke onset. If treatment with beta-blockers was started before stroke onset and no other information on use after stroke onset was specified, we interpreted this as that the therapy had continued during the first 7 days after stroke as well. Treatment with beta-blockers occurred as monotherapy or in combination with other antihypertensive agents. The comparison group consisted of patients without beta-blocker therapy, with or without other antihypertensive drugs. For an article to be included, at least one of the three studied outcomes (mortality, functional outcome, and infection up to the first 12 months from stroke onset) had to be presented.

\section{Data Extraction}

The following data were extracted from the included articles: the name of the first author, year of publication, study population, study design, number of patients in the intervention and the comparison group, mortality rate, number of patients with poor functional outcome in each group, infection rate and odds ratio adjusted for age and severity, if available. If the outcome of interest was evaluated at more than one time point, data were extracted at a time point as early as possible, but of at least 1 month from ictus. If infections were reported both in total (all diagnoses lumped together) and specifically for the different diagnoses (pneumonia, etc.), the total number was extracted. 


\section{Outcome Measures}

The three outcome measures were mortality rate, functional outcome measured with Barthel Index (BI) or modified Rankin Scale (mRS), and rate of infection, respectively. Functional outcome was dichotomized into the two categories "good" and "poor". Since guidelines for dichotomizing $\mathrm{mRS}$ and $\mathrm{BI}$ are lacking, we opted for the commonly used cut-off of $\mathrm{mRS}>3$ or $\mathrm{BI} \leq 60 / 100$ (or $\leq$ $12 / 20$, depending on the scoring type) to define poor functional outcome. ${ }^{20}$ For each outcome, adjusted odds ratio (OR) or adjusted rate ratio (RR) with $95 \%$ confidence interval (CI) for age and stroke severity was also recorded. Also, the unadjusted OR and $95 \%$ CI for the univariate analysis was recorded or a crude OR was calculated using the numbers extracted from the study.

\section{Statistical Analysis}

Since age and stroke severity are strong confounders, especially in observational studies, analyses for all three outcomes were primarily performed with ORs adjusted for these factors. However, since studies presenting OR adjusted for age and stroke severity were relatively few, additional analyses also including unadjusted/crude OR from studies lacking adjusted ORs were performed as a complement. In the pooled meta-analysis, $\mathrm{RR}$ and $\mathrm{OR}$ were used interchangeably depending on which one was used in most of the studies, since the two ratios approximate one another when the event rate is small. ${ }^{21}$ Statistical heterogeneity was investigated using $\mathrm{I}^{2}$ statistics. ${ }^{22} \mathrm{I}^{2}>30 \%$ was considered moderate heterogeneity while $\mathrm{I}^{2}>50 \%$ was considered substantial heterogeneity. $\mathrm{P}$ value of heterogeneity $<0.05$ was considered statistically significant. ${ }^{23}$ When the studies presented obvious heterogeneity, a random effect model was used; otherwise, a fixed-effect model was applied. ${ }^{24}$ The risk of publication bias was assessed using Egger's test and visually inspected using funnel plots. ${ }^{25}$ Sensitivity analyses were performed using the leave-one-out (LOO) method and sources of heterogeneity were demonstrated using the Baujat plot. Finally, a cumulative meta-analysis was performed to examine the reliability of the results. All the analyses were performed in Stata 16.1 (StataCorp LLC, College Station, Texas, USA) and a two-sided $p$ value of $<0.05$ was considered statistically significant unless otherwise specified.

\section{Quality Assessment}

The methodological qualities of the selected articles were assessed using checklists provided by the Swedish Agency For Health Technology Assessment And Assessment Of Social Services, one for assessing randomized clinical trials and one for observational studies. ${ }^{26}$

\section{Results}

\section{Literature Search}

The search results yielded a total number of 5142 studies. After the removal of duplicates, 3953 titles remained. After screening these studies by reading titles and abstracts, 29 were chosen for full-text assessment. The search was further expanded by searching the reference lists of these 29 studies, by searching for studies that had cited these 29 studies in Scopus, and by using the "similar articles" lists in PubMed, and additional 34 studies eligible for full-text assessment were found. This resulted in a total of 63 studies, of which 43 were excluded after full-text assessment (Figure 1).

Of the remaining 20 studies, two ${ }^{27,28}$ were based on the same database. The overlap of the study populations was considerable therefore they were handled as one study. One study ${ }^{29}$ presented data separately on ischemic and hemorrhagic stroke and was therefore treated as two separate studies and will from hereon be referred to as such. If data on mortality and functional outcome were available at more than one time point, we chose to extract data from the study that was more in accordance with our time point definition. In total, 18 of the 20 studies presented adjusted and/or unadjusted ORs for the analysis of mortality, ${ }^{27,29-44}$ 11 for functional outcome, ${ }^{28,32-34,38-42,45,46}$ and 11 for infection. ${ }^{28,30,34-38,40-43}$

\section{Study Characteristics}

Two of the 20 included studies were randomized controlled trials, ${ }^{31,33}$ one was an exploratory analysis based on a randomized controlled trial, ${ }^{40}$ and 16 were observational studies. ${ }^{27-30,32,34-39,41-46}$ For a detailed outline of the studies and study characteristics, see Table S5. Selection bias was deemed low in 15 of the studies ${ }^{27-31,35-44}$ and moderate in $5 . .^{32-34,45,46}$ Treatment bias was low in 14 studies $^{27-31,33-38,40-42}$ and moderate in $6^{32,39,43-46}$ The assessment bias was low in $16^{27-31,33,35-44}$ and moderate in $4 .^{32,34,45,46}$ The fall-out bias was low in $4^{29,38,40,42}$ and moderate in $4 .{ }^{28,31,33}$ The 


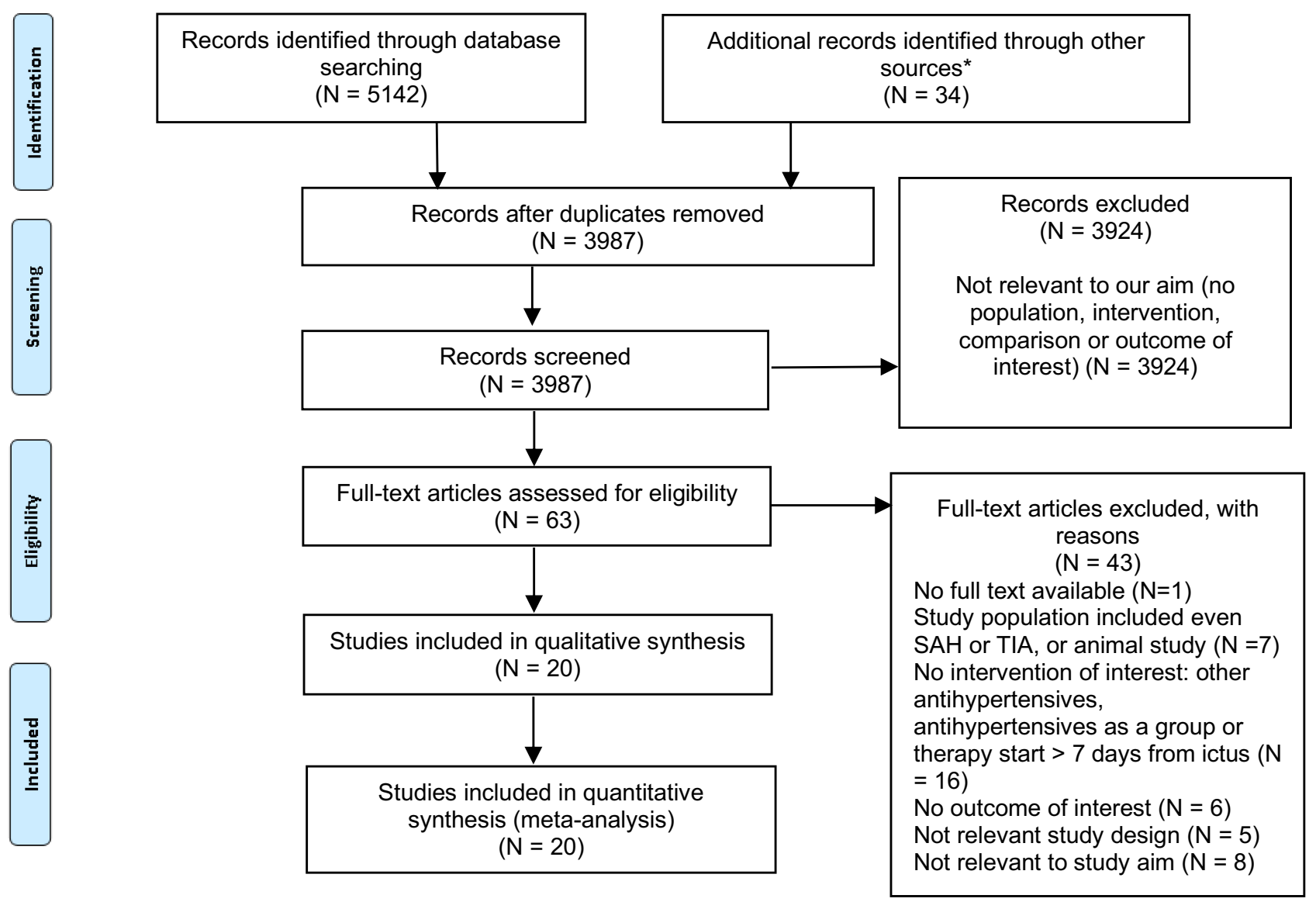

Figure I PRISMA flow diagram of study selection. ${ }^{21}$

reporting bias was low in $18^{27-29,32,33,35-47}$ and moderate in $2 .^{30,34}$ The conflict of interest bias was low in all the studies. Ten of the studies $29,30,35-38,40-42,44$ were evaluated to have a low risk for bias and $10^{27,28,32-34,39,43,45-47}$ had a moderate risk for bias. See Table S6 for details on the quality assessment. On average, the quality assessments indicated that the risk of bias was moderate.

\section{Outcomes}

\section{Mortality}

Neither the meta-analysis based on age- and severityadjusted ORs (pooled RR: 0.90; 95\% CI 0.65-1.24; Figure 2) nor the corresponding meta-analysis on both adjusted and unadjusted ORs (pooled RR: 0.94; 95\% CI 0.79-1.12; Figure 3) showed a significant effect of betablockers on mortality. The 10 studies in the adjusted OR analysis included a total of 17,001 individuals, and the 18 studies in both the adjusted and unadjusted OR analysis included a total of 116,540 individuals. The studies proved to be heterogeneous $\left(\mathrm{I}^{2}=89.1 \%\right.$ and $95.9 \%$ for the analysis on adjusted and both adjusted and unadjusted ORs; $\mathrm{p}=$ 0.000 for both) and a random-effects model was used for both analyses. Egger's test showed weak $(\mathrm{p}=0.084$; funnel plot in Figure S1) and significant ( $\mathrm{p}=0.014$; funnel plot in Figure S2) risk for publication bias based on the studies in the analysis of the adjusted and both adjusted and unadjusted ORs, respectively. In the sensitivity analyses for the analysis on both adjusted and unadjusted ORs, one study ${ }^{37}$ was identified as having a relatively large influence on heterogeneity (Figures S3 and S4). However, it did not substantially change the pooled results (Figures S5 and S6). Although the cumulative metaanalysis showed significant variation in the pooled results (Figures S7 and S8), none of the results were statistically significant and the overall conclusion remained unchanged.

\section{Functional Outcome}

In analogy with the mortality analyses, neither the functional outcome meta-analysis based on age- and severity- 


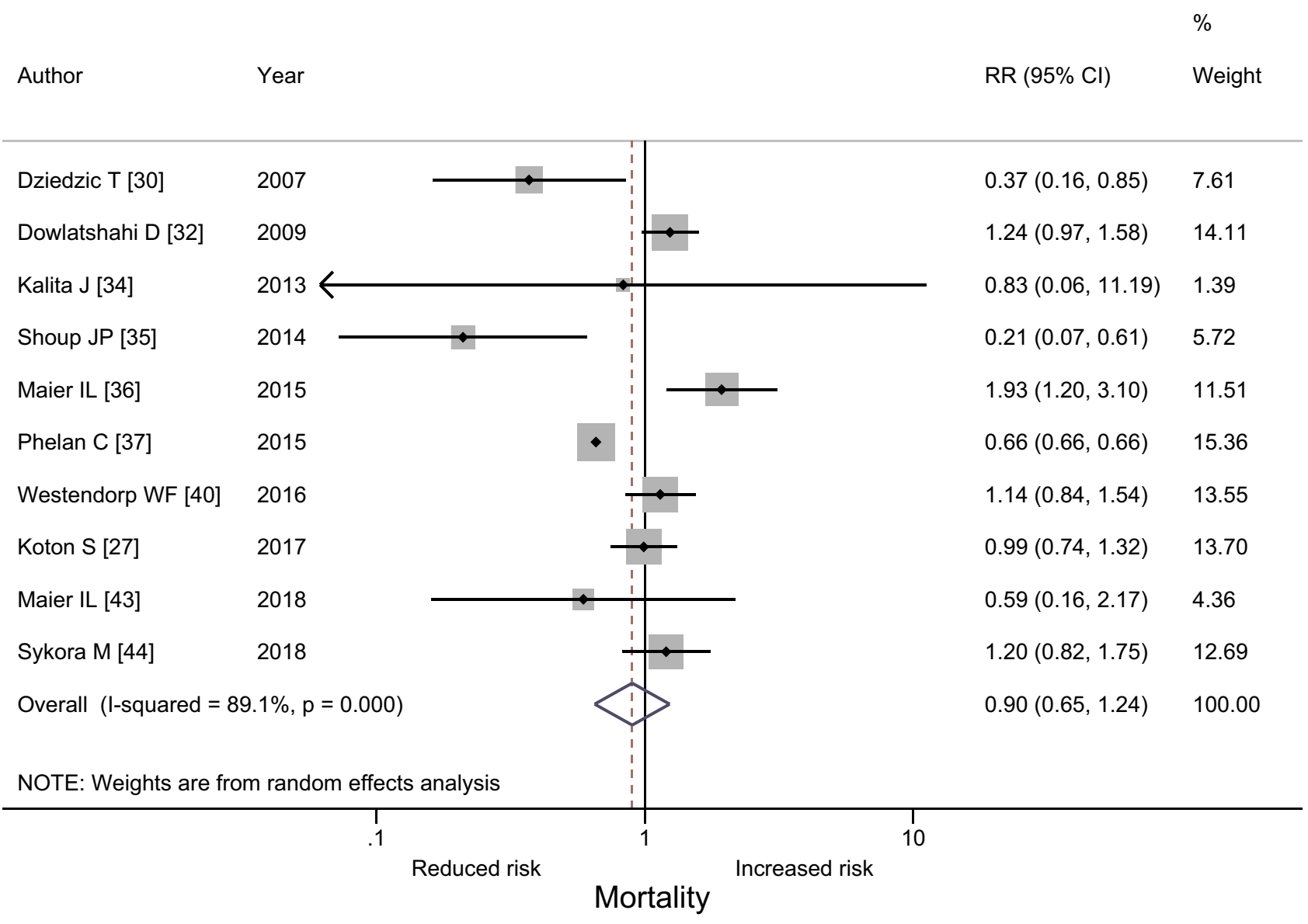

Figure 2 Forest plot analyzing the association between beta-blocker treatment and post-stroke mortality, presenting adjusted (for stroke severity and age) rate ratio (RR) and $95 \%$ confidence interval $(\mathrm{Cl})$.

adjusted ORs (pooled OR: 1.04; 95\% CI 0.92-1.18; Figure 4) nor the corresponding meta-analysis on both adjusted and unadjusted ORs (pooled OR: 0.99; 95\% CI 0.91-1.08; Figure 5) showed a significant effect of beta-blockers. The three studies in the adjusted OR analysis included a total of 9,038 individuals, and the 11 studies in the adjusted and unadjusted OR analysis included a total of 19,122 individuals. The studies were homogenous, $\left(\mathrm{I}^{2}=0.0 \%, \mathrm{p}=0.780\right.$ and $\mathrm{I}^{2}=7.3 \%, \mathrm{p}=0.375$ for the analysis on adjusted and both adjusted and unadjusted ORs) and a fixed-effects model was used for both analyses. Egger's test showed no risk for publication bias $(\mathrm{p}=0.674$; funnel plot in $\underline{\text { Figure S9 }}$ and $\mathrm{p}=0.296$; funnel plot in Figure S10 based on the studies in the analysis of the adjusted and both adjusted and unadjusted ORs, respectively). In the sensitivity analysis for the adjusted ORs there were only three studies included (Figures S11 and $\underline{\mathrm{S} 12}$ ). In the sensitivity analysis for both the adjusted and unadjusted ORs two studies stood out as outliers, ${ }^{34,39}$ affecting the overall heterogeneity contribution (Figures S13 and S14). No significant variation was found in the cumulative analyses (all pooled ORs are around 1.0) (Figures S15 and S16).

\section{Infection}

The results were similar for the infection outcome as for mortality and functional outcomes. Neither the metaanalysis based on age- and severity-adjusted ORs (pooled OR: 1.26; 95\% CI 0.79-2.01; Figure 6) nor the corresponding meta-analysis on both adjusted and unadjusted ORs (pooled OR: 1.03; 95\% CI 0.75-1.42; Figure 7) showed a significant effect of beta-blockers on the rate of infections. The two studies in the adjusted OR analysis included a total of 3,158 individuals, and the 11 studies in both the adjusted and unadjusted OR analysis included a total of 14,305 individuals. The studies were heterogeneous, $\left(\mathrm{I}^{2}=81.6 \%\right.$, 


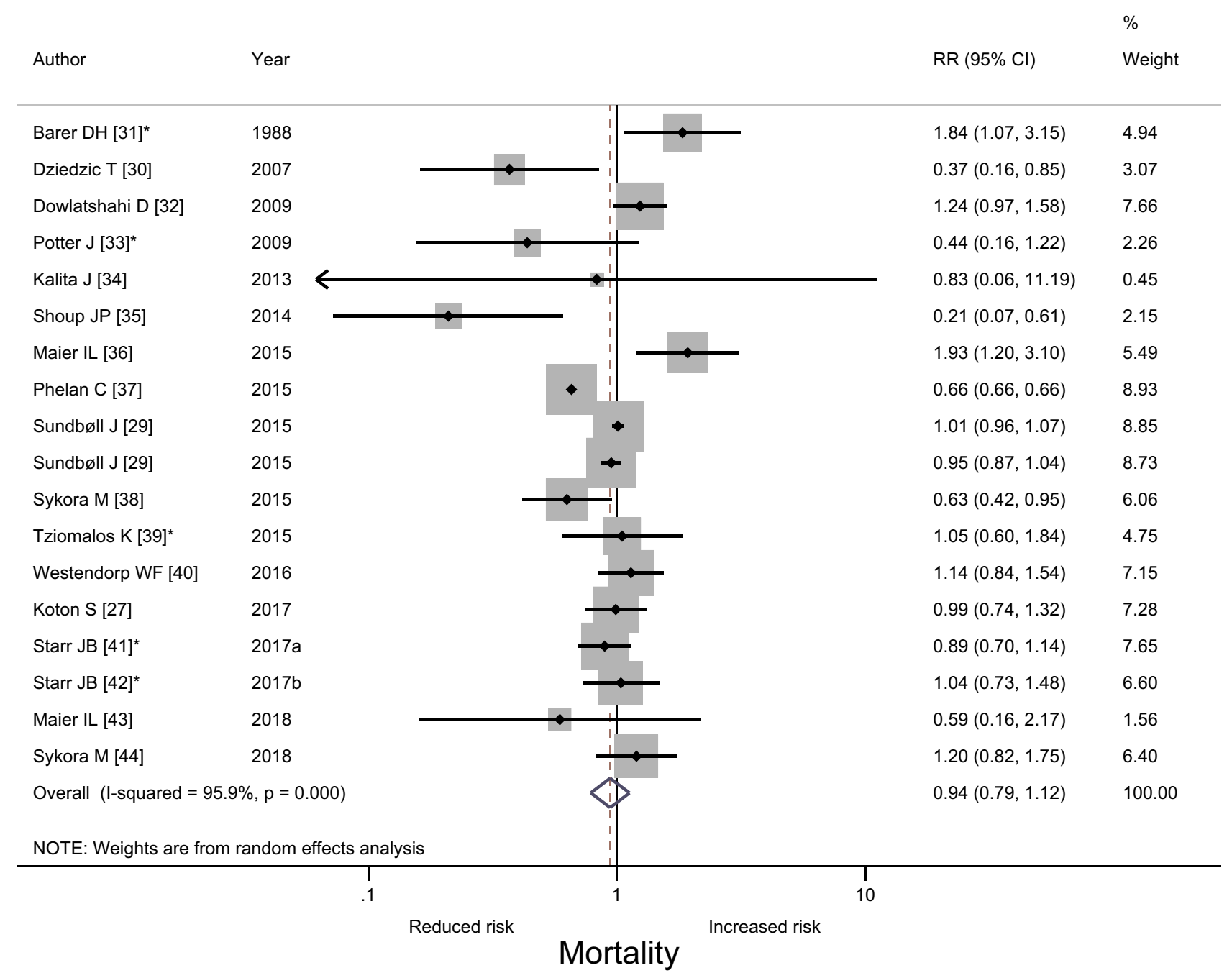

Figure 3 Forest plot analyzing the association between beta-blocker treatment and post-stroke mortality, presenting rate ratio (RR) and $95 \%$ confidence interval (Cl). For study author with *, crude RR was used.

$\mathrm{p}=0.020$ and $\mathrm{I}^{2}=77.9 \%, \mathrm{p}=0.000$ for the analysis of adjusted and both adjusted and unadjusted ORs) and a random-effects model was used for both analyses. Egger's test showed no risk for publication bias $(p=0.224$; funnel plot in Figure S17) based on the studies in the analysis of both the adjusted and unadjusted ORs. Eggers's test could not be performed based on the studies in the analysis of the adjusted ORs, because the studies were too few (funnel plot presented in Figure S18). In the sensitivity analysis for the adjusted ORs, there were only two studies included, with Westendorp et a ${ }^{40}$ contributing heavily to the overall heterogeneity (Figure S19). Due to the small sample of studies, the LOO analysis was inconclusive (Figure S20). In the sensitivity analyses for both the adjusted and unadjusted ORs, four studies $^{28,34,35,38}$ stood out as heavier contributors of overall heterogeneity, but with Kalita et $\mathrm{al}^{34}$ as the strongest outlier (Figures S21 and S22). Although the cumulative metaanalyses showed variation in the pooled results, none of the results was statistically significant (Figures S23 and S24).

For GRADE:ing and overview of the results, see Table S7.

\section{Discussion}

The pooled analyses showed no significant association between beta-blocker therapy and any of the three outcomes, neither with data adjusted for stroke severity and age nor with both adjusted and unadjusted data. This is, to the best of our knowledge, the first meta-analysis 


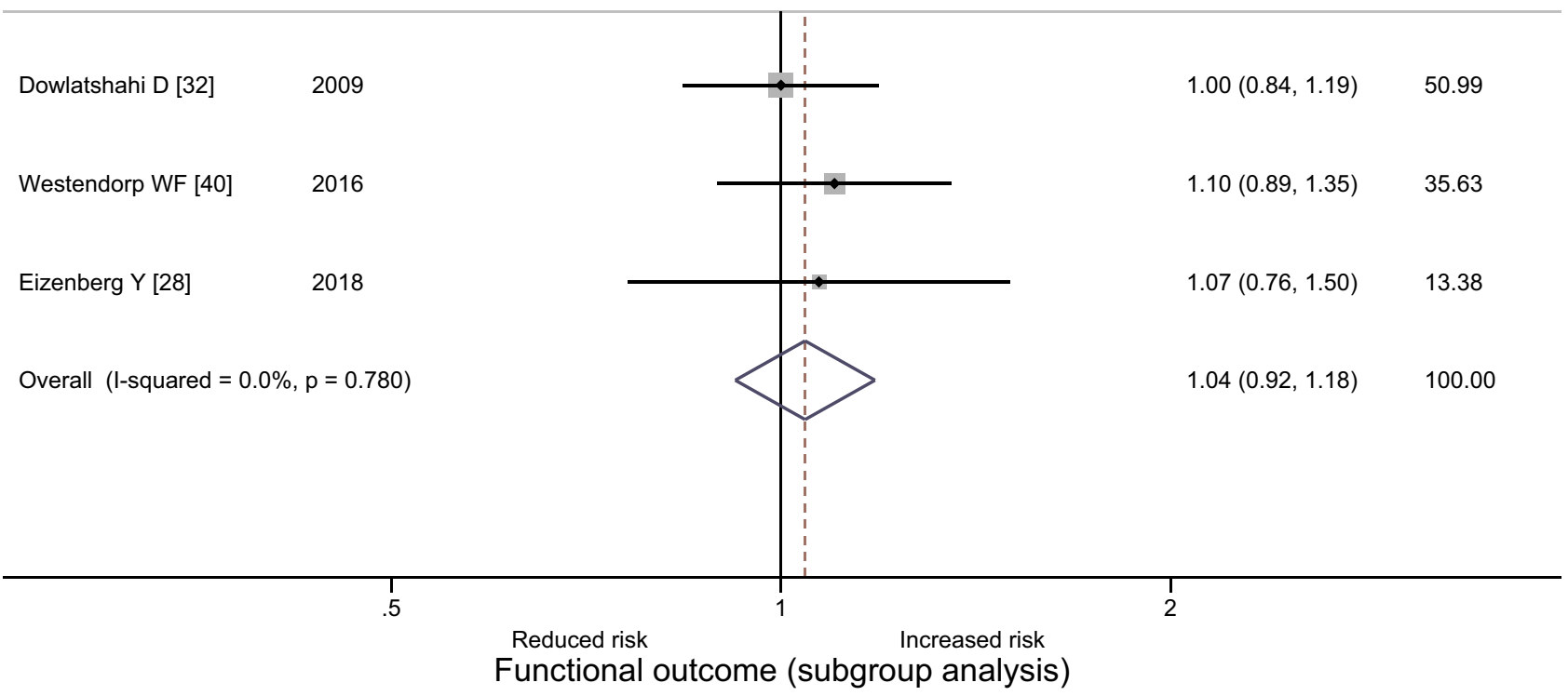

Figure 4 Forest plot analyzing the association between beta-blocker treatment and functional outcome after an acute stroke, presenting adjusted (for stroke severity and age) odds ratio (OR) and $95 \%$ confidence interval (Cl).

evaluating the association between beta-blockers and stroke outcome.

The current meta-analysis is mainly based on observational studies, which calls for caution when assessing the results. However, if it is assumed that the patients treated with beta-blockers were in all other aspects comparable to the patients not receiving beta-blockers, the results strongly suggest that beta-blockers have no valuable effect in the acute stage after stroke. However, since such comparability between the groups is not warranted, it is also possible that an eventual effect of the beta-blockers was obscured by differences between treated and untreated patients. Receiving beta-blockers could be a marker for vascular disease burden, predisposing for a more severe stroke or for vascular complications, ${ }^{32}$ which could be out-weighed by protective effects of the beta-blockers. Indeed, one of the studies indicating harm from beta-blocker therapy after stroke found that when eliminating cardiovascular causes of death after a stroke, treatment with betablockers was no longer associated with increased mortality. ${ }^{32}$ This effect of beta-blockers being a marker for vascular disease burden would probably be strongest in patients that were already on beta-blocker therapy at the time of stroke, which was the case in all but seven of the included studies. In other words, it is possible that beta-blockers would have been beneficial if the groups had been equal at baseline. However, counterbalancing this argument is the fact that the two included randomized clinical trials did not show a benefit from beta-blocker therapy after stroke. Barer et $\mathrm{al}^{31}$ compared treatment with atenolol $50 \mathrm{mg}$ or slow-release propranolol $80 \mathrm{mg}$ to placebo in 302 beta-blocker naïve patients with hemispheric stroke, excluding patients with pre-existing major disability or contraindications to beta-blockers (for example, baseline heart rate of less than 56 beats per minute or systolic blood pressure less than $100 \mathrm{mmHg}$ ). The treatment was started within 48 hours after ictus and was administered orally on a daily basis for 3 weeks or until discharge. The trial was planned to be performed in two stages, but the trial was stopped after the first stage because treatment 


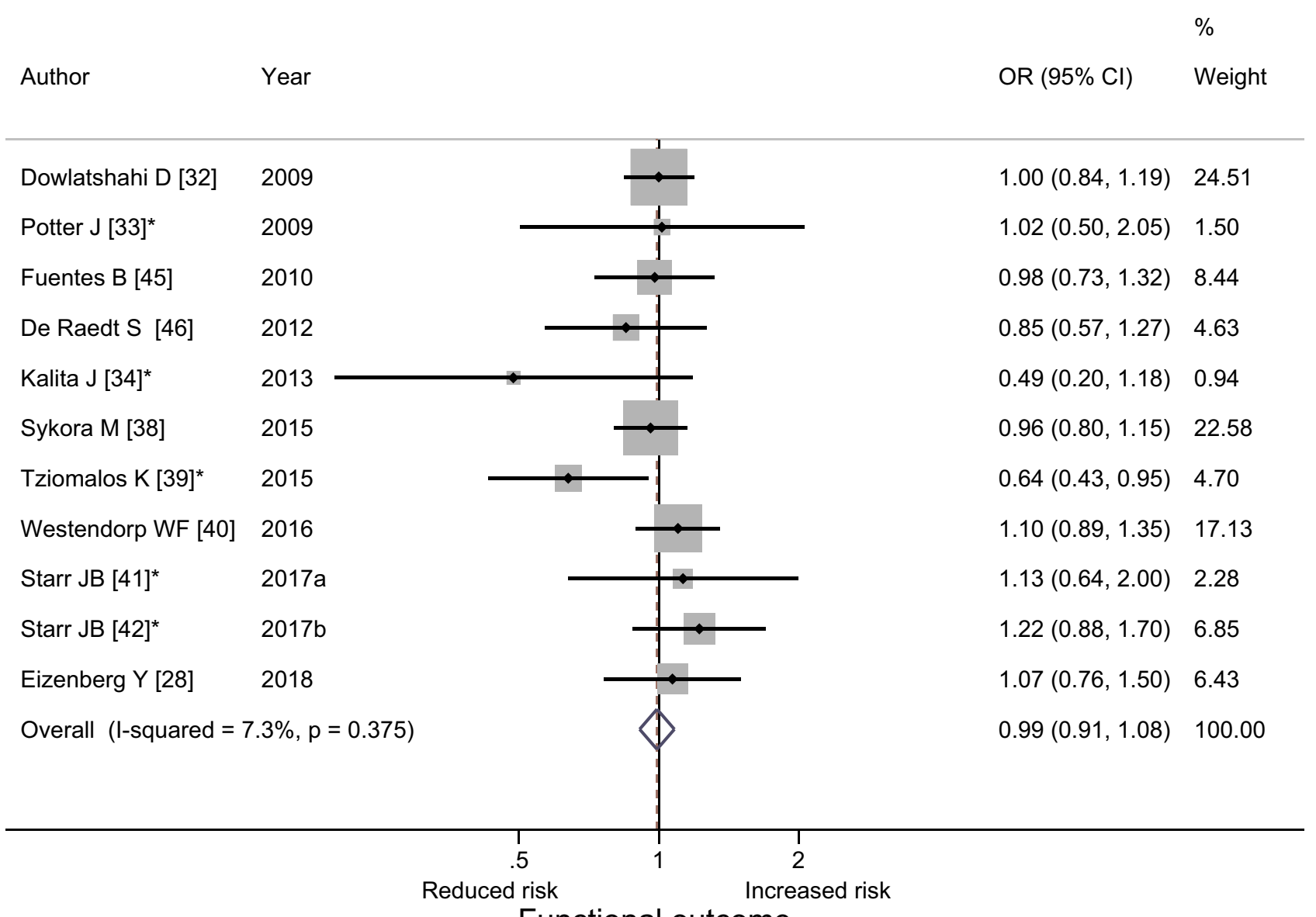

Functional outcome

Figure 5 Forest plot analyzing the association between beta-blocker treatment and functional outcome after stroke, presenting odds ratio (OR) and $95 \%$ confidence interval (Cl). For studies with *, crude OR was used.

seemed to increase mortality, especially in the elderly. This effect was small and after adjustment for confounders between the randomized groups, it diminished even further. No protective benefits with treatment with betablockers could be seen. In the other randomized controlled trial, Potter et $\mathrm{al}^{33}$ compared $5 \mathrm{mg}$ lisinopril or $50 \mathrm{mg}$ labetalol to placebo in patients with ischemic or hemorrhagic stroke and a systolic blood pressure above $160 \mathrm{mmHg}$. Patients with a blood pressure over 200/120 $\mathrm{mmHg}$ and intracerebral hemorrhage were excluded, as were subjects with impaired consciousness or contraindications to the drugs. Treatment was started within 36 hours from stroke onset and was administered orally in non-dysphagic patients, while dysphagic patients had a corresponding regime of intravenous lisinopril or intravenous labetalol, respectively. During the first 24 hours, blood pressure was monitored closely, and additional doses were given for patients not reaching a systolic blood pressure of 145-155 mmHg. Thereafter, the patients received fixed daily doses until 2 weeks after the stroke. One-hundred and twenty-six of the 179 patients completed the full 14-day treatment, with slightly more withdrawals in the lisinopril (18 patients) and labetalol groups (16 patients) than in the placebo group (11 patients). No significant differences were found in neither mortality nor dependency at 2 weeks between the patients who did and did not receive beta-blockers.

Although there are studies on animal models supporting the beneficial effect of beta-blockers after an acute stroke, our meta-analysis could not corroborate those findings. One possible explanation is that blood pressure suppression may have a negative effect in the acute phase of stroke because it may encumber adequate blood perfusion 


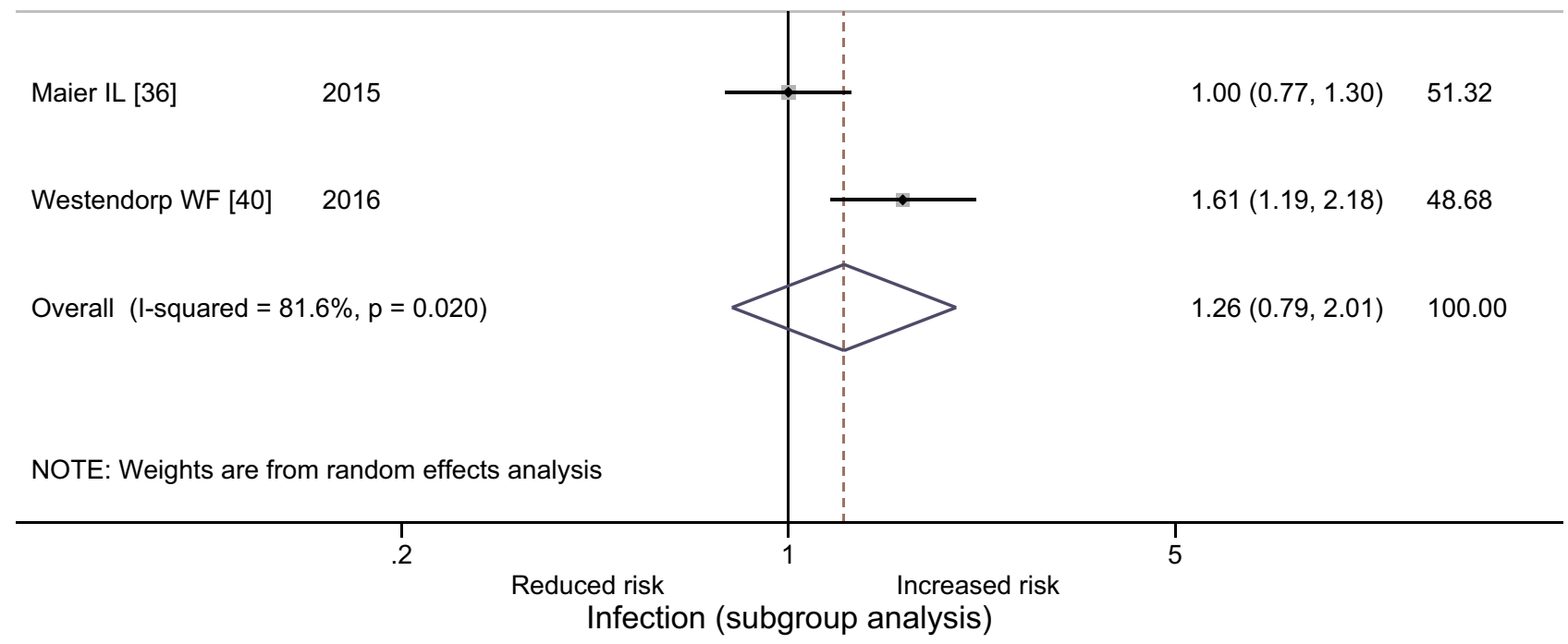

Figure 6 Forest plot analyzing the association between beta-blocker treatment and post-stroke infections, presenting adjusted (for stroke severity and age) odds ratio (OR) and $95 \%$ confidence interval $(\mathrm{Cl})$.

of the cerebral tissue. Another possibility is that betablockers' effect on the immune system may not be as desirable as has been suggested. It is indeed conceivable that the stroke-induced immunosuppression may be harmful by increasing the risk of bacterial infections. However, the ischemic process leads to necrosis and blood-brain barrier disruption, exposing intracellular proteins for the adaptive immune system and thereby risking autoimmune reactions. ${ }^{48}$ It may be the case that the immunosuppression following stroke is not at all excessive, but rather an inherent mechanism trying to balance the risk of bacterial infections against the risk of autoimmune reactions.

The current study's main limitation is that it is based on other, previously published, studies, making it impossible to control for medication bias or potential confounders like socioeconomic status, access to medical care or treatment indication. Beta-blocker therapy was loosely defined and consisted of a highly heterogeneous group of medications with varying doses and treatment length, and often combined with other antihypertensive medications. Subjects in the comparison group had often other antihypertensive medication which can also influence stroke outcome. Because it's unclear if beta-blockers have any classspecific properties, comparing them with other antihypertensives may complicate the interpretation of the results. Further, the included articles rarely contained information regarding the discontinuation of beta-blocker treatment. In fact, the only studies accounting for this were the two randomized controlled trials. ${ }^{31,33}$ Since the withdrawal of beta-blockers could affect stroke outcome, not being able to account for this factor is a weakness of the metaanalysis. Our analysis is based on a small pool of eligible studies most of which are observational studies, thus our results and conclusions may be less reliable and of lesser quality than desired. Also, the Egger analyses indicated a moderate risk for publication bias for some of the outcomes. This means that there may be research that was not accessible for our pooled analysis.

In conclusion, our meta-analysis failed to show an association between beta-blocker therapy and post-stroke 


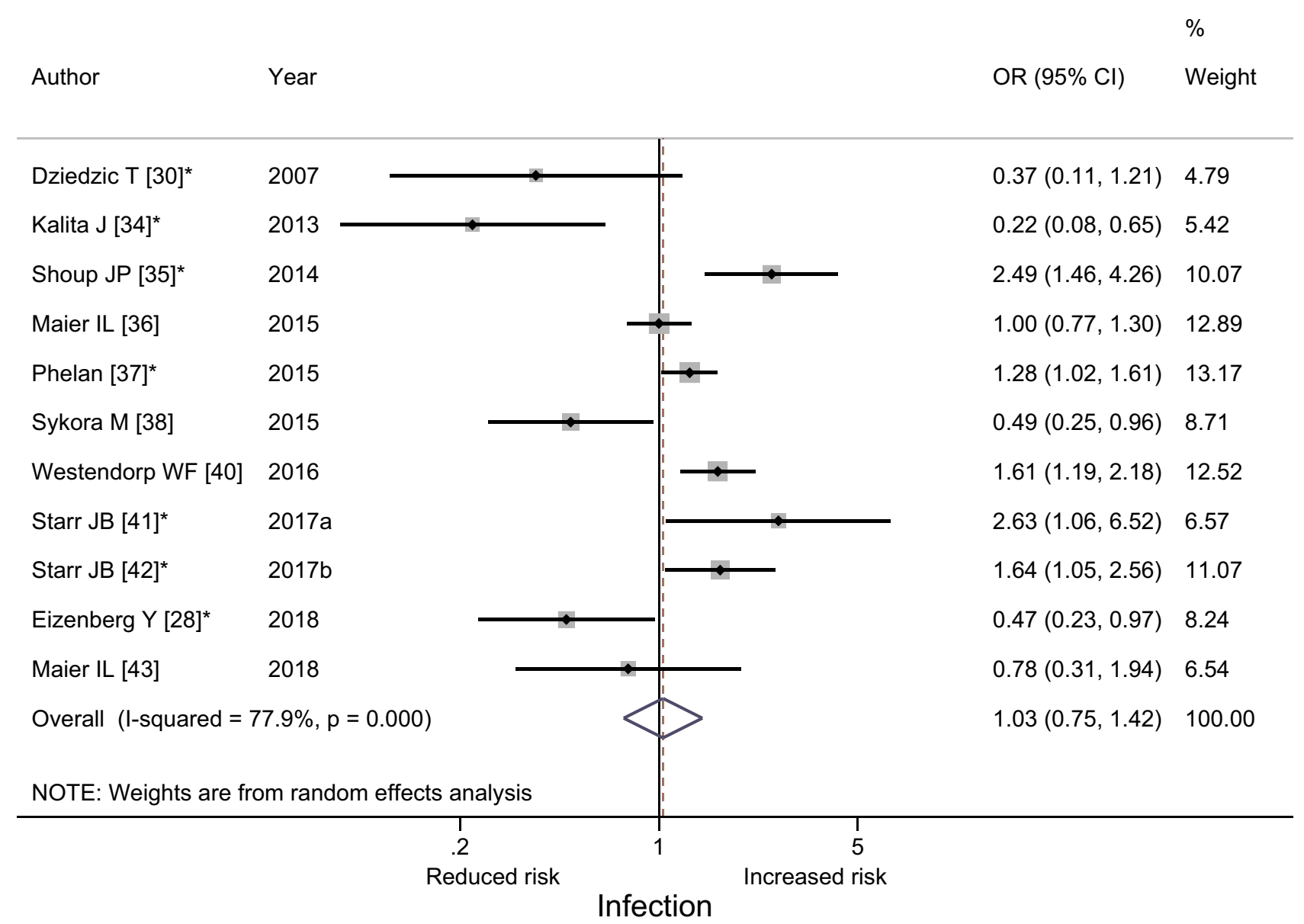

Figure 7 Forest plot analyzing the association between beta-blocker treatment and post-stroke infections, presenting odds ratio (OR) and $95 \%$ confidence interval (Cl). For study author with *, crude OR was used.

mortality, functional outcome and infections, irrespective of whether or not the data were adjusted for age and stroke severity.

\section{Author Contributions}

All authors made a significant contribution to the work reported, whether that was in the conception, study design, execution, acquisition of data, analysis and interpretation, or in all these areas; took part in drafting, revising or critically reviewing the article; gave final approval of the version to be published; have agreed on the journal to which the article has been submitted; and agree to be accountable for all aspects of the work.

\section{Disclosure}

Dr Jakob O Ström reports personal fees from Bayer AB, outside the submitted work. The authors report no other potential conflicts of interest for this work.

\section{References}

1. World Health Organisation. The top 10 causes of death [Internet]. Geneva: World Health Organization; 2018 [cited November 6, 2018]. Available from: https://www.who.int/news-room/fact-sheets/detail/thetop-10-causes-of-death.

2. Sander D, Winbeck K, Klingelhöfer J, Etgen T, Conrad B. Prognostic relevance of pathological sympathetic activation after acute thromboembolic stroke. Neurology. 2001;57:833-838. doi:10.1212/WNL.57.5.833

3. Xiong L, Tian G, Leung H, et al. Autonomic dysfunction predicts clinical outcomes after acute ischemic stroke: a prospective observational study. Stroke. 2018;49:215-218. doi:10.1161/STROKEAHA.117.019312

4. Barron SA, Rogovski Z, Hemli J. Autonomic consequences of cerebral hemisphere infarction. Stroke. 1994;25:113-116. doi:10.1161/01. STR.25.1.113

5. Sykora M, Diedler J, Poli S, et al. Autonomic shift and increased susceptibility to infections after acute intracerebral hemorrhage. Stroke. 2011;42:1218-1223. doi:10.1161/STROKEAHA.110.604637

6. Chamorro A, Amaro S, Vargas M, et al. Catecholamines, infection, and death in acute ischemic stroke. J Neurol Sci. 2007;252:29-35. doi:10.1016/j.jns.2006.10.001

7. Prass K, Meisel C, Höflich C, et al. Stroke-induced immunodeficiency promotes spontaneous bacterial infections and is mediated by sympathetic activation reversal by poststroke $\mathrm{T}$ helper cell Type 1-like immunostimulation. J Exp Med. 2003;198:725-736. doi:10.1084/ jem.20021098 
8. Savitz SI, Erhardt JA, Anthony JV, et al. The novel beta-blocker, carvedilol, provides neuroprotection in transient focal stroke. J Cereb Blood Flow Metab. 2000;20:1197-1204. doi:10.1097/00004647200008000-00005

9. Lysko PG, Lysko KA, Yue TL, Webb CL, Gu JL, Feuerstein G. Neuroprotective effects of carvedilol, a new antihypertensive agent, in cultured rat cerebellar neurons and in gerbil global brain ischemia Stroke. 1992;23:1630-5; discussion 1635-1636.

10. Heeba GH, El-Hanafy AA. Nebivolol regulates eNOS and iNOS expressions and alleviates oxidative stress in cerebral ischemia/reperfusion injury in rats. Life Sci. 2012;90:388-395. doi:10.1016/j. lfs.2011.12.001

11. Standefer M, Little JR. Improved neurological outcome in experimental focal cerebral ischemia treated with propranolol. Neurosurgery. 1986;18:136-140. doi:10.1227/00006123-198602000-00003

12. Laowattana S, Oppenheimer SM. Protective effects of beta-blockers in cerebrovascular disease. Neurology. 2007;68:509-514. doi:10.1212/01.wnl.0000253186.23949.fd

13. Kinnander G, Viitanen M, Asplund K. Beta-adrenergic blockade after stroke. A preliminary closed cohort study. Stroke. 1987;18:240-243. doi:10.1161/01.STR.18.1.240

14. Dowlatshahi D, Fang J, Kawaja M, Hakim A. Use of calcium channel blockers after stroke is not associated with poor outcome: a cohort from the registry of the Canadian stroke network. $J$ Neurol. 2006;253:1478-1483. doi:10.1007/s00415-006-0249-1

15. Yu AYX, Keezer MR, Zhu B, Wolfson C, Côté R. Pre-stroke use of antihypertensives, antiplatelets, or statins and early ischemic stroke outcomes. Cerebrovasc Dis. 2009;27:398-402. doi:10.1159/000207444

16. Heeley EL, Wei JW, Wang J-G, et al. Comparative effects of antihypertensive drugs on stroke outcome in China. Int J Stroke. 2014;9 (Suppl A100):113-118. doi:10.1111/ijs.12330

17. Tziomalos K, Giampatzis V, Bouziana SD, et al. Effect of antihypertensive treatment on the long-term outcome of patients discharged after acute ischemic stroke. Clin Exp Hypertens. 2017;39:246-250. doi:10.1080/10641963.2016.1246561

18. Aerden L, van Raak E, Boreas A, Kessels A, Lodder J. Common drugs in acute ischemic stroke and their influence on outcome: an observational study in 1013 stroke patients. Clin Neuropharmacol. 2008;31:74-79. doi:10.1097/WNF.0b013e31811ec142

19. Liberati A, Altman DG, Tetzlaff J, et al. The PRISMA statement for reporting systematic reviews and meta-analyses of studies that evaluate health care interventions: explanation and elaboration. PLoS Med. 2009;6:e1000100. doi:10.1371/journal.pmed.1000100

20. Sulter G, Steen C, De Keyser J. Use of the barthel index and modified rankin scale in acute stroke trials. Stroke. 1999;30(8):1538-1541. doi:10.1161/01.STR.30.8.1538

21. Zhang J, Yu KF. What's the relative risk? A method of correcting the odds ratio in cohort studies of common outcomes. JAMA. 1998;280:1690-1691. doi:10.1001/jama.280.19.1690

22. Higgins JPT, Thompson SG, Deeks JJ, Altman DG. Measuring inconsistency in meta-analyses. BMJ. 2003;327:557-560. doi:10.1136/bmj.327.7414.557

23. Higgins J, Green S. Cochrane handbook for systematic reviews of interventions version 5.1.0 [Internet]. The Cochrane Collaboration; 2011 [updated March, 2011; cited December 3, 2018]. Available from: http://handbook-5-1.cochrane.org/.

24. DerSimonian R, Laird N. Meta-analysis in clinical trials. Control Clin Trials. 1986;7:177-188. doi:10.1016/0197-2456(86)90046-2

25. Egger M, Davey Smith G, Schneider M, Minder C. Bias in meta-analysis detected by a simple, graphical test. $B M J$. 1997;315:629-634. doi:10.1136/bmj.315.7109.629

26. Swedish Agency For Health Technology Assessment And Assessment Of Social Services. Assessment of methods in health care A handbook [Internet]. 2018 [cited December 2, 2018]. Available from: https://www.sbu.se/contentassets/76adf07e270c48efa f67e3b560b7c59c/eng_metodboken.pdf.
27. Koton S, Tanne D, Grossman E. Prestroke treatment with beta-blockers for hypertension is not associated with severity and poor outcome in patients with ischemic stroke: data from a national stroke registry. $J$ Hypertens. 2017;35:870-876. doi:10.1097/ HJH.0000000000001218

28. Eizenberg Y, Grossman E, Tanne D, Koton S. Pre admission treatment with Beta-blockers in hypertensive patients with acute stroke and 3-month outcome-Data from a national stroke registry. J Clin Hypertens. 2018;20:568-572. doi:10.1111/jch.13211

29. Sundbøll J, Schmidt M, Horváth-Puhó E, et al. Impact of preadmission treatment with calcium channel blockers or beta blockers on short-term mortality after stroke: a nationwide cohort study. BMC Neurol. 2015;15:24. doi:10.1186/s12883-015-0279-3

30. Dziedzic T, Slowik A, Pera J, Szczudlik A. Beta-blockers reduce the risk of early death in ischemic stroke. J Neurol Sci. 2007;252:53-56. doi:10.1016/j.jns.2006.10.007

31. Barer DH, Cruickshank JM, Ebrahim SB, Mitchell JR. Low dose beta blockade in acute stroke ('BEST' trial): an evaluation. $\mathrm{Br}$ Med $J$. 1988;296:737-741. doi:10.1136/bmj.296.6624.737

32. Dowlatshahi D, Hakim A, Fang J, Sharma M. Pre admission antithrombotics are associated with improved outcomes following ischaemic stroke: a cohort from the Registry of the Canadian Stroke Network. Int $J$ Stroke. 2009;4:328-334. doi:10.1111/j.17474949.2009.00331.x

33. Potter J, Mistri A, Brodie F, et al. Controlling hypertension and hypotension immediately post stroke (CHHIPS)-a randomised controlled trial. Health Technol Assess. 2009;13:1-73. doi:10.3310/ hta13090

34. Kalita J, Misra UK, Kumar B. Is $\beta$-blocker (atenolol) a preferred antihypertensive in acute intracerebral hemorrhage? Neurol Sci. 2013;34:1099-1104. doi:10.1007/s10072-012-1210-y

35. Shoup JP, Winkler J, Czap A, et al. $\beta$-Blockers associated with no class-specific survival benefit in acute intracerebral hemorrhage. J Neurol Sci. 2014;336:127-131. doi:10.1016/j.jns.2013.10.022

36. Maier IL, Karch A, Mikolajczyk R, Bähr M, Liman J. Effect of betablocker therapy on the risk of infections and death after acute strokea historical cohort study. PLoS One. 2015;10:e0116836. doi:10.1371/ journal.pone. 0116836

37. Phelan C, Alaigh V, Fortunato G, Staff I, Sansing L. Effect of $\beta$ adrenergic antagonists on in-hospital mortality after ischemic stroke. J Stroke Cerebrovasc Dis. 2015;24:1998-2004. doi:10.1016/j. jstrokecerebrovasdis.2015.04.035

38. Sykora M, Siarnik P, Diedler J; VISTA Acute Collaborators. $\beta$ blockers, pneumonia, and outcome after ischemic stroke: evidence from virtual international stroke trials archive. Stroke. 2015;46:1269-1274. doi:10.1161/STROKEAHA.114.008260

39. Tziomalos K, Giampatzis V, Bouziana SD, et al. Effects of different classes of antihypertensive agents on the outcome of acute ischemic stroke. J Clin Hypertens. 2015;17:275-280. doi:10.1111/ jch. 12498

40. Westendorp WF, Vermeij J-D, Brouwer MC, et al. Pre-stroke use of beta-blockers does not lower post-stroke infection rate: an exploratory analysis of the preventive antibiotics in stroke study. Cerebrovasc Dis. 2016;42:506-511. doi:10.1159/000450926

41. Starr JB, Tirschwell DL, Becker KJ. Labetalol use is associated with increased in-hospital infection compared with nicardipine use in intracerebral hemorrhage. Stroke. 2017;48:2693-2698. doi:10.1161/ STROKEAHA.117.017230

42. Starr JB, Tirschwell DL, Becker KJ. Increased infections with $\beta$ blocker use in ischemic stroke, a $\beta 2$-receptor mediated process? Neurol Sci. 2017;38:967-974. doi:10.1007/s10072-017-2877-x

43. Maier IL, Becker JC, Leyhe JR, et al. Influence of beta-blocker therapy on the risk of infections and death in patients at high risk for stroke induced immunodepression. PLoS One. 2018;13:e0196174. doi:10.1371/journal.pone. 0196174 
44. Sykora M, Putaala J, Meretoja A, Tatlisumak T, Strbian D. Betablocker therapy is not associated with mortality after intracerebral hemorrhage. Acta Neurol Scand. 2018;137:105-108. doi:10.1111/ ane. 12817

45. Fuentes B, Fernández-Domínguez J, Ortega-Casarrubios MA, SanJosé B, Martínez-Sánchez P, Díez-Tejedor E. Treatment with angiotensin receptor blockers before stroke could exert a favourable effect in acute cerebral infarction. J Hypertens. 2010;28:575-581. doi:10.1097/HJH.0b013e3283350f50

46. De Raedt S, Haentjens P, De Smedt A, et al. Pre-stroke use of beta-blockers does not affect ischaemic stroke severity and outcome. Eur J Neurol. 2012;19:234-240. doi:10.1111/j.14681331.2011.03475.x
47. Barer DH. The influence of visual and tactile inattention on predictions for recovery from acute stroke. QJM. 1990;74:21-32.

48. Klehmet J, Hoffmann S, Walter G, Meisel C, Meisel A. Stroke induces specific alteration of $\mathrm{T}$ memory compartment controlling auto-reactive CNS antigen-specific T cell responses. J Neurol Sci. 2016;368:77-83. doi:10.1016/j.jns.2016.06.039
Clinical Epidemiology

\section{Publish your work in this journal}

Clinical Epidemiology is an international, peer-reviewed, open access, online journal focusing on disease and drug epidemiology, identification of risk factors and screening procedures to develop optimal preventative initiatives and programs. Specific topics include: diagnosis, prognosis, treatment, screening, prevention, risk factor modification,

Submit your manuscript here: https://www.dovepress.com/clinical-epidemiology-journal
Dovepress

systematic reviews, risk \& safety of medical interventions, epidemiology \& biostatistical methods, and evaluation of guidelines, translational medicine, health policies \& economic evaluations. The manuscript management system is completely online and includes a very quick and fair peer-review system, which is all easy to use. 